\title{
Estudio comparativo de herramientas software libre para la Gestión de Procesos de Negocio
}

\author{
$\square$ Edinson RaÚl SERRANo-Abreo ${ }^{1}$ \\ HERNÁN CAMILO CASTEllanos-GRANADOS ${ }^{2}$
}

\section{Resumen}

Análisis comparativo de plataformas de Gestión de Procesos de Negocio libres (BPM), estableció y expuso las funciones deseadas para una herramienta BPM, en pro de facilitar su selección para abordar la gestión, automatización y mejora continua de los procesos en las organizaciones. Durante el análisis se seleccionó un grupo de plataformas BPM libres y se estableció un grupo de parámetros de inclusión para evaluarlas y seleccionar dos plataformas BPM de tal grupo. Las mejor calificadas fueron BonitaBPM Community Edition y ProcessMaker Open Source. Posteriormente se estableció un marco de 85 parámetros de evaluación, que aplica para evaluar cualquier plataforma BPM, no solo libres. Luego se estableció el mecanismo de puntuación y las formas de evaluación, y se ejecutó la evaluación comparativa de las dos plataformas libres seleccionadas, dando como la mejor calificada a BonitaBPM, en la cual se implementó un prototipo de un proceso de solicitud de crédito con buenos resultados.

Palabras clave: BPM, BPMS, iBPMS, BonitaBPM, ProcessMaker, Comparación parámetros BPM.

\section{Comparative study of free software tools for Business Process Management}

\section{Abstract}

Comparative Analysis of Free Business Process Management (BPM) platforms, established and exposed the desired functions for a BPM tool, in order to facilitate its selection to address the management, automation and continuous improvement of the processes in the Organizations. During the analysis selected a group of free BPM platforms and

1 Hernández Gómez Constructora, Bucaramanga, Colombia.

2 Universidad Industrial de Santander UIS, Bucaramanga, Colombia.

Autor de correspondencia: Serrano Abreo, E.R. (Edinson Raúl): Hernández Gómez Constructora, C.C. Cabecera Iv Etapa L 401 Bucaramanga, Colombia. Código postal: 680003. Teléfono: 3144304762. Correo electrónico: edinsonraul@gmail.com
Historia del artículo:

Artículo recibido: 19-VIII-2017/ Aprobado: 14-I-2019

Disponible online: 3 de febrero de 2019

Discusión abierta hasta octubre de 2020 
established a group of inclusion parameters to evaluate them and select two BPM platforms from that group. The best rated were BonitaBPM Community Edition and ProcessMaker Open Source. Subsequently a framework of 85 evaluation parameters was established, which is applied to evaluate any BPM platform, not only free. Then, the scoring mechanism and evaluation forms were established, and the benchmarking of the two free platforms selected was carried out, with BonitaBPM as the best rated, in which a prototype of a credit application process was implemented, with successful results.

Keywords: BPM, BPMS, iBPMS, BonitaBPM, ProcessMaker, BPM Parameter Comparison.

\section{Estudo comparativo de ferramentas de software livre para Gestão de Processos de Negocios}

\section{Resumo}

Análise comparativa de platforms Livres de estão de processos de negocios, estabeleceu e expôs as funções desejadas para uma ferramenta BPM, A fim de facilitar sua seleção para abordar a gestão, automação e melhoria contínua dos processos nas Organizações. Durante a análise, um grupo de plataformas BPM LIVRES foi selecionado e um grupo de parâmetros de incluso foi estabelecida para avaliá-los e selecionar duas plataformas BPM desse grupo. Os melhores classificados foram BonitaBPM Community Edition and ProcessMaker Open Source. Logo após, foi estabelecida uma estrutura de 85 parâmetros de avaliação, que é aplicada para avaliar qualquer plataforma BPM, não apenas livres, e o avaliação comparativa foi realizado nas duas plataformas livres selecionadas, com o BonitaBPM como o melhor classificado, no qual foi implementado um protótipo de um processo de pedido de crédito com bons resultados.

Palavras-chave: BPM, BPMS, iBPMS, BonitaBPM, ProcessMaker, Comparação de parâmetros BPM.

\section{Introducción}

En un entorno global cada vez más dinámico, que exige adaptación rápida y mejora continua para mantener a las organizaciones competitivas, y esto solo se logra mejorando la respuesta al cambio, la eficacia y eficiencia de los procesos que las soportan. La tendencia hoy es la Gestión de Procesos de Negocio (BPM: Business Process Management), una disciplina de administración que trata los procesos de negocios como activos. Supone que los objetivos organizacionales se pueden lograr a través de definición, ingeniería, control y dedicación a la mejora continua de los procesos de negocio(Moore, C; Saxena, Raju; Lee, 2013).

El interés por BPM ha crecido exponencialmente, según Gartner en el año 2015 las organiza- ciones gastaron alrededor de 2.7 billones utilizando BPM como una plataforma para la digitalización de procesos de negocio, uno de los casos de uso BPM más buscados en la actualidad(Dunie, Rob;Schulte, 2015). Ahora en Latinoamérica y específicamente en Colombia existen casos de éxito referenciados por el club BPM(“Club-BPM," n.d.) y BPM Latino(“BPMLatino.com," n.d.), entidades dedicadas a la promulgación, impulso y formación de BPM en España y Latinoamérica. Casos como el Ministerio de las TIC (MINTIC), Escuela Colombiana de Ingeniería, Promotora de Café Colombia, Allianz Colombia, Gas Natural Colombia, Ministerio del Interior Colombia, entre otras. Esto dejo claro que BPM es una disciplina vigente en el mundo y en Colombia, principalmente en grandes compañías como las referenciadas, sin embargo, su adopción requiere de la alineación de 
estrategia empresarial con la metodología y una inversión subyacente.

Partiendo de la base que el costo de proyectos BPM puede ser bastante alto como lo sugirió un análisis reciente de BPTrends, una consultora especializada en procesos de negocio a más de 100 afiliados alrededor del mundo relacionados con BPM, que arrojó que el $52 \%$ de las organizaciones que representan han gastado hasta $\$ 500.000$ dólares en BPM y el resto cantidades superiores en BPM(Harmon, 2016). Por tal motivo fue necesario indagar una alternativa que redujera los costos en cuanto a uno de los factores de mayor gasto, el software BPM, en pro de que facilitar la adopción.

Identificada la problemática y partiendo de la base que Forrester (Miers, 2013) ha identificado 52 BPMS en el mercado, el objetivo fue realizar una evaluación comparativa de dos BPMS, que por las razones de importancia de costo expuestas debían ser libres o tener una versión libre y ser representativos en este segmento, de tal forma que el trabajo aportara claridad sobre los parámetros que permitan la comparación y faciliten la selección de un BPMS que soporte de la mejor manera posible una iniciativa BPM. Con base en el objetivo se definió la pregunta de investigación de la siguiente manera:

¿De qué manera se compara y se selecciona entre dos BPMS libres en el mercado?, problema que a su vez se desprende en otros subproblemas:

1. ¿Cómo se seleccionan los dos BPMS libres a comparar si existen muchos en el mercado?

2. ¿Cuáles son los parámetros sobre los que se deben comparar las herramientas?

3. ¿Cómo comprobar las prestaciones de BPMS, identificando ventajas/desventajas para abordar una iniciativa BPM?

Problemas semejantes se han abordado por otros autores (Hill, Jones, Schulte, \& Sinur, 2012) (Miers, 2013)(Schulte \& Cantara, 2014)(Dunie, Rob;Schulte, 2015)(Sinur \& Hill, 2010), pero muy enfocados en la evaluación del proveedor, más que del mismo software BPMS y sin especificación clara sobre los parámetros utilizados para la evaluación, y sobre escenarios muy modernos sobre los que BPMS libres pueden no operar aun. Expuestos los argumentos y partiendo de la premisa de que el BPM es un concepto en constante evolución, se realizó un estudio sobre el tema planteado que permitiera consolidar los avances de otras publicaciones relacionadas de diferentes autores, tomarlas como base y complementarlas para definir y establecer los parámetros relevantes para evaluar un BPMS y conocer los BPMS libres más representativos a la fecha del análisis, evaluarlos comparativamente y desplegar un prototipo de proceso de negocio en la mejor evaluada.

\section{Marco teórico}

La Gestión de Procesos de Negocio (BPM) envuelve una serie de conceptos, por lo que se le dio inicio al proceso definiendo algunos de ellos antes de entrar en materia:

- Proceso de negocio: Definido como un conjunto de actividades impulsadas por eventos y que ejecutadas en cierta secuencia crean valor para el cliente(Histpass, 2014).

- $\quad$ Ocurrencia de proceso: Llamada caso de negocio, y se define como una colección de tareas, acciones, procesos y contenido en apoyo a un objetivo de negocio(Kitson, Ravisanskar, \& Soudamini, 2012).

- BPM: Es un enfoque sistemático para identificar, levantar, documentar, diseñar, ejecutar, medir y controlar procesos manuales y automatizados con la finalidad de lograr a través de sus resultados en forma consistente los objetivos de negocio que están alineados con la estrategia de la organización, promueve el mejoramiento, innovación y gestión de procesos de principio a fin, que determinan los resultados de negocio, crean valor para el cliente y posibilitan el logro de objetivos de negocio con mayor agilidad(Binner, $\mathrm{H}$;Bariff, $\mathrm{M}$;Benedict, T; Champlin, B; Downing, B; Franzen, 2009). 
Luego se profundizo en BPM, enfocados en su soporte basado en la gestión de procesos de negocio en diferentes fases que se comportan como un ciclo de vida BPM, descritas así(Hitpass, 2014) (Brocke, J; Rosemann, 2010):

- Diseño y Modelado: Métodos para identificar y conceptualizar la actualidad(as is) y el futuro (to be) de los procesos de negocio, además de los métodos de análisis de procesos. En esta etapa se lleva a cabo la identificación, levantamiento de la información acerca del flujo de trabajo del proceso, políticas de negocio, procedimientos, que se documentan en un modelo de procesos.

- Implementación y Ejecución: Métodos que ayudan a transformar los modelos de procesos en especificaciones de procesos de negocio ejecutables. Abarca implementación técnica y adaptaciones organizacionales requeridas.

- Monitorización y Medición: Se refiere a los métodos que proporcionan dirección para la recolección y dirección de los datos relacionados a los procesos, relacionados al control continuo de procesos como riesgos, o medidas de rendimiento de procesos como tiempo, costo y calidad.

- Optimización e Innovación: Se enfoca en todos los métodos que facilitan el desarrollo de procesos de negocio mejorados. Incluye actividades que soportan mejora de procesos, innovación de procesos, mejor uso de personas, datos y sistemas a través de la identificación de debilidades que se traducen en mejoras.

Las funciones que permiten cubrir las fases BPM se encuentran en herramientas software especializadas llamadas Sistemas de Gestión de Procesos de Negocio (BPMS), que Gartner describe como una colección integrada de tecnologías de software que posibilitan la transparencia en los procesos, y así mejorar su gestión, también como el trabajo en el proceso, que utilizan modelos de proceso explícitos para en tiempo de ejecución coordinar (orquestar) la interacción entre personas, sistemas e información como otros aspectos de trabajo de principio a fin con una experiencia de usuario unificada dentro del BPMS(Sinur \& Hill, 2010). Estos han evolucionado a lo que Gartner llama Sistemas de Gestión de Procesos de Negocio Inteligente (iBPMS), que define como un BPMS tradicional con funcionalidad extendida para soportar Operaciones de Negocio Inteligente (IBO), tales como analítica de negocio en tiempo real, Procesamiento de Eventos Complejos (CEP), medios sociales para soportar comportamiento social y colaboración, tecnología expandida para soportar los crecientes requerimientos de movilidad (Hill et al., 2012).

Las definiciones de BPMS/iBPMS dadas referencian grosso modo algunas de las características/ funcionalidades esperadas en una herramienta software de tal tipo, claro está, cada una posible gracias a una serie de elementos software que las componen, que Gartner (Dunie, Rob; Schulte, 2015) expone de los iBPMS, evolución de los BPMS (Tabla 1).

\section{Marco metodológico}

\section{A. Método de Investigación}

Se inició con análisis detallado de la literatura en el área de Gestión de Procesos de Negocio y la comparación de BPMS de diferentes fuentes y analistas de tecnología como Gartner, Forrester(Sinur \& Hill, 2010)(Hill et al., 2012)(Miers, 2013)(Dunie, Rob;Schulte, 2015), elaborando un análisis detallado, que posibilitó consolidar y comprender los conceptos BPM, identificar los parámetros a utilizar para comparar los BPMS y filtrar un listado de BPMS libres representativos en el mercado. Subsecuentemente se diseñó un método de filtrado consistente en parámetros (criterios) enfocados en el éxito de los proveedores/herramientas para evaluarlos/calificarlos y obtener los dos BPMS libres a comparar. Después se consolidaron los parámetros de comparación de los BPMS obtenidos en el estado del arte y literatura BPM de forma que se obtuvo un marco de evaluación/comparación sólido. 
TABLA 1. ELEMENTOS ESENCIALES DE UN IBPMS

FUENTE: (DUNIE, ROB; SCHULTE, 2015)

\begin{tabular}{|c|c|}
\hline Elemento & Descripción \\
\hline $\begin{array}{l}\text { Motor de Orquestación } \\
\text { de Procesos }\end{array}$ & $\begin{array}{l}\text { Coordina interacción de todos los tipos de actores, personas, dispositivos y sistemas informáticos para } \\
\text { flujos estructurados y no estructurados, compatible con la gestión de casos y procesos dinámicos. Gestiona } \\
\text { procesos de corta y larga duración, cambios de estado de los recursos coordinados. Ajusta prioridades y } \\
\text { orden de ejecución de las instancias de procesos. }\end{array}$ \\
\hline $\begin{array}{l}\text { Entorno Composición } \\
\text { Grafico basado en } \\
\text { Modelos }\end{array}$ & $\begin{array}{l}\text { Soporta herramientas de creación/desarrollo, y en tiempo de ejecución aplicaciones compuestas } \\
\text { heterogéneamente, flujos de proceso y opcionalmente reglas. Cubre la validación del modelo del proceso, } \\
\text { verificando dominios, advertencias y patrones inconsistentes. Soporta interfaz de usuario para construir } \\
\text { portales, interfaces de usuario móviles y enriquecidas para el cliente. }\end{array}$ \\
\hline $\begin{array}{l}\text { Entorno de Interacción } \\
\text { de Contenido }\end{array}$ & $\begin{array}{l}\text { Gestiona o integra con herramientas Gestoras de Contenido Empresarial (Enterprise Content Management: } \\
\text { ECM) para crear, leer, enrutar, y actualizar el contenido gestionado documentos imágenes, video, audio. }\end{array}$ \\
\hline Interacciones humanas & $\begin{array}{l}\text { Soporta mesas de trabajo personalizadas para los participantes en los procesos (en base a factores tales } \\
\text { como Rol, las preferencias y los permisos de acceso), y proporciona acceso interactivo a las tareas, el } \\
\text { contenido y otros recursos. Ofrece capacidades de colaboración para ayudar a las personas a intercambiar } \\
\text { datos e ideas acerca de un proceso de una manera flexible y controlada por el usuario. }\end{array}$ \\
\hline Analítica sobredemanda & $\begin{array}{l}\text { Proporciona servicios analíticos que se ejecutan a partir de los requerimientos de las personas, } \\
\text { aplicaciones o dispositivos para tomar una decisión informada: } \\
\text { - Simulación y Optimización de Procesos: Servicios que mejoran el modelo del proceso de negocio o la } \\
\text { forma como está siendo ejecutado. } \\
\text { - El análisis predictivo: Servicios que utilizan modelos matemáticos y la lógica para calcular } \\
\text { acontecimientos futuros probables u otros hechos no conocidos. }\end{array}$ \\
\hline $\begin{array}{l}\text { Analítica } \\
\text { Activa(continua) }\end{array}$ & $\begin{array}{l}\text { Monitoreo Actividades de Negocio (Business Activity Monitoring: BAM) e Inteligencia de Procesos } \\
\text { suministra información instantánea, inteligencia continua para conducir el trabajo: } \\
\text { - Monitoreo, métricas de procesos (interacciones y recursos). } \\
\text { - } \quad \text { Alertas de amenazas y oportunidades, disparadores automáticos para responder a ellas. } \\
\text { - } \quad \text { Registro de eventos de procesos. } \\
\text { - } \quad \text { Adaptadores para capturar eventos desde fuera del motor de orquestación de procesos. }\end{array}$ \\
\hline $\begin{array}{l}\text { Procesamiento de } \\
\text { Reglas de Negocio }\end{array}$ & $\begin{array}{l}\text { Gestiona y ejecuta las reglas que representan políticas de negocio. Lleva a cabo un razonamiento basado } \\
\text { en software que infiere consecuencias lógicas a partir de un conjunto de hechos o axiomas. Gestiona y } \\
\text { ejecuta las reglas que representan políticas de negocio. }\end{array}$ \\
\hline Conectividad & $\begin{array}{l}\text { Soporta conexión con aplicaciones comerciales estándar y soporta protocolos y estándares de conexión } \\
\text { como: } \\
\text { - } \quad \text { Protocolo de Transferencia de Hipertexto (Hypertext Transfer Protocol: HTTP). } \\
\text { - } \quad \text { Arquitectura REST (Transferencia de Estado Representacional: Representational State Transfer). } \\
\text { - } \quad \text { Protocolo de Acceso a Objetos Simples (Simple Object Access Protocol: SOAP). } \\
\text { - } \quad \text { Lenguaje de descripción de servicios web (Web Services Description Language: WSDL). } \\
\text { - Conectividad abierta de Base de Datos (Open DataBase Connectivity: ODBC). } \\
\text { - Conectividad Base Datos Java (Java DataBase Connectivity: JDBC). }\end{array}$ \\
\hline $\begin{array}{l}\text { Gestión y } \\
\text { Administración }\end{array}$ & $\begin{array}{l}\text { - Configuración, administración de plataforma iBPMS y los artefactos de aplicación, control de } \\
\text { versiones, seguridad aplicación, roles, usuarios, funciones. } \\
\text { - Gestión y Monitoreo del rendimiento de los procesos y sus componentes asociados, así como } \\
\text { auditoria. }\end{array}$ \\
\hline Registro/Repositorio & $\begin{array}{l}\text { Gestión y almacenamiento de objetos y metadatos de procesos en tiempo de diseño y ejecución. } \\
\text { Capacidades de reportes, control de versiones, controles de seguridad. }\end{array}$ \\
\hline
\end{tabular}


Posteriormente se estableció un prototipo de un proceso de solicitud de crédito desde la perspectiva BPM tomado de una entidad real con créditos al público, teniendo presente que BPM no estaba implementado en tal organización y que el proceso solo estaba sistematizado en una parte. Definidos los BPMS libres y un marco de evaluación/comparación se realizó un análisis de cada uno respecto a un grupo filtrado de los parámetros de tal marco, seleccionando para evaluar solo los viables y relevantes en el contexto de investigación. La evaluación de las dos plataformas BPM seleccionadas en tales parámetros se llevó a cabo definiendo unos métodos de evaluación y puntuación, obteniendo una evaluación comparativa. Finalmente en el BPMS mejor calificado se implementó el prototipo del proceso de solicitud de crédito establecido previamente.

\section{Resultados de la investigación}

Se inició por la selección de un grupo de plataformas BPM (BPMS/iBPMS) libres, para después definir un grupo de parámetros de inclusión en el análisis para evaluarlos en cada plataforma BPM del grupo y seleccionar las dos mejor calificadas para luego establecer un marco de parámetros de evaluación/comparación que permitiera evaluar en detalle las capacidades deseadas para un BPMS y aplicarlas a las dos plataformas BPM libres seleccionadas, para a la mejor calificada de las dos utilizarla para desplegar un prototipo de un proceso de solicitud de crédito, y concluir.

\section{A. Selección de las Dos Plataformas} BPM Para Su Comparación/Evaluación

La selección de las plataformas BPM a analizadas se desarrolló en dos pasos detallados así:

1) Definición de los Parámetros de Inclusión/ Selección de las Dos Plataformas BPM libres

A partir de los parámetros de inclusión de las publicaciones analizadas en el estado del arte, principalmente de las firmas Gartner y Forrester acerca de BPMS y iBPMS se cruzaron y se seleccionaron los considerados relevantes en el contexto de análisis de BPMS libres y teniendo en cuenta que el enfoque estaba en los temas técnicos de tales plataformas BPM libres y no en un análisis de mercado BPM consolidando el resultado en la Tabla 2.

2) Selección de Dos Plataformas BPM Libres Aplicando los Parámetros de Inclusión Se seleccionó un grupo de plataformas BPM evaluando el parámetro número 1 de inclusión (Tabla 2), a partir del mismo análisis de la literatura, y con bases en las menciones a las plataformas BPM que se clasifican como BPMS en las publicaciones de Forrester (Miers, 2013) y Gartner (Sinur \& Hill, 2010) (Hill et al., 2012) (Schulte \& Cantara, 2014) (Dunie, Rob; Schulte, 2015) y que referencian a cinco proveedores de BPMS que son libres o tienen una versión libre. El resultado de la evaluación con la puntuación asignada se muestra en Tabla 3.

Adicionalmente se consideró un BPMS libre bastante conocido en la comunidad libre y no referenciado en las publicaciones analizadas llamado Activiti ("Activiti," n.d.). Filtrados los proveedores BPMS se revisaron sus versiones para garantizar que tuvieran una versión libre que cubriera el ciclo de vida BPM completo. Las consideraciones para descartar BPMS fueron:

- Bizagi se excluyó porque la ejecución de procesos en su plataforma solo es libre en ambiente de pruebas, y el enfoque fue plataformas BPM libres en producción.

- Intalio se excluyó porque a pesar de tener una versión Community referenciada en las publicaciones analizadas, no fue posible a la fecha encontrarla ni siquiera en su página corporativa, por lo que se presume ya no dan soporte para la versión libre.

- $\quad$ Red Hat ofrece dos plataformas BPM, jBPM y JBoss BPM Suite, esta última fue descartada del análisis porque solo es libre para uso por parte de desarrolladores individuales de forma individual y en ambiente de pruebas. 
TABLA 2. PARÁMETROS DE INCLUSIÓN/SELECCIÓN DE PLATAFORMAS BPM CONSIDERADAS EN EL ESTUDIO

FUENTE: ELABORACIÓN PROPIA

\begin{tabular}{|c|c|c|c|}
\hline Núm. & Parámetro & $\begin{array}{c}\text { Máxima } \\
\text { Ponderación }\end{array}$ & Observación \\
\hline 1 & $\begin{array}{l}\text { Reconocimiento plataforma BPM } \\
\text { entre las publicaciones de analistas } \\
\text { reconocidos }\end{array}$ & 10 & $\begin{array}{l}\text { Plataforma BPM referenciado en publicaciones } \\
\text { analizadas en estado del arte (Gartner y Forrester). }\end{array}$ \\
\hline 2 & $\begin{array}{l}\text { Cantidad Mundial descargas } \\
\text { plataforma BPM en repositorios de } \\
\text { libre descarga }\end{array}$ & 5 & $\begin{array}{l}\text { Se analizaron la cantidad mundial de descargas de la } \\
\text { plataforma BPM desde sourceforge. }\end{array}$ \\
\hline 3 & $\begin{array}{l}\text { Porcentaje descargas plataforma BPM } \\
\text { en Colombia respecto a la mundial }\end{array}$ & 5 & $\begin{array}{l}\text { Cantidad de descargas de la plataforma BPM en } \\
\text { sourceforge en Colombia respecto a la mundial de la } \\
\text { misma plataforma, esto midió de su impacto cuanto } \\
\text { repercusión en Colombia. }\end{array}$ \\
\hline 4 & $\begin{array}{l}\text { Industrias en las que la Plataforma } \\
\text { del proveedor BPM pueda ser } \\
\text { utilizada. }\end{array}$ & 5 & $\begin{array}{l}\text { Se indagó tipos de industrias en las que plataformas } \\
\text { BPM pudieran utilizarse, evaluando paginas } \\
\text { corporativas BPMS. }\end{array}$ \\
\hline 5 & $\begin{array}{l}\text { Nivel de importancia de los clientes } \\
\text { utilizan plataformas del Proveedor } \\
\text { BPM }\end{array}$ & 5 & $\begin{array}{l}\text { Nivel de importancia de la plataforma BPM utilizada } \\
\text { por clientes empresariales se consultó en páginas } \\
\text { empresariales. }\end{array}$ \\
\hline 6 & $\begin{array}{l}\text { Nivel de fortaleza de la comunidad } \\
\text { alrededor de la plataforma BPM }\end{array}$ & 10 & $\begin{array}{l}\text { Estimación del tamaño de comunidad alrededor de } \\
\text { plataforma como foros, usuarios inscritos, u otros. }\end{array}$ \\
\hline 7 & $\begin{array}{l}\text { Plataforma BPM libre en el todo el } \\
\text { ciclo BPM y una versión propietaria }\end{array}$ & 10 & $\begin{array}{l}\text { Plataforma BPM con versión libre y versión propietaria } \\
\text { que tenga prestaciones evolucionadas. }\end{array}$ \\
\hline
\end{tabular}

TABLA 3. PROVEEDORES BPMS CON VERSIÓN LIBRE REFERENCIADAS POR GARTNER Y FORRESTER

FUENTE: ELABORACIÓN PROPIA

\begin{tabular}{|l|l|c|c}
\multicolumn{1}{c|}{$\begin{array}{c}\text { Proveedor } \\
\text { Plataforma BPM }\end{array}$} & Referencias que lo clasifican como un BPMS & $\begin{array}{c}\text { Cantidad } \\
\text { referencias }\end{array}$ & $\begin{array}{c}\text { Puntuación } \\
\text { parámetro 1 }\end{array}$ \\
\hline BonitaSoft & Forrester 2013, Gartner 2012 & 2 & 4 \\
\hline 2016Colosa(ProcessMaker) & Forrester 2013 & 1 & 2 \\
\hline Bizagi & Forrester 2013, Gartner 2010, 2012 & 3 & 6 \\
\hline Intalio & Forrester 2013, Gartner 2010, 2012 & 3 & 6 \\
\hline Red Hat & Forrester 2013, Gartner 2012, 2014, 2015 & 4 & 8 \\
\hline
\end{tabular}

Definidos los BPMS de los cuales se evaluarían sus versiones libres (BonitaBPM, ProcessMaker, jBPM, Activiti) contra los parámetros de inclusión (Tabla 2) para seleccionar los dos que se utilizarían en la siguiente fase de comparación, y calificada la categoría uno de inclusión, se procedió a evaluar el resto de parámetros. La evaluación de parámetros 2 y 3 (Figura 1) se hizo con base a la cantidad de descargas desde un repositorio de libre descarga (sourceforge) entre julio 1 de 2015 y julio 10 de 2016, dando como resultado las descargas mundiales (circulo externo) y en Colombia (circulo interno) junto a sus respectivos porcentajes, incluido el porcentaje de descargas en Colombia respecto al total de cada plataforma en el mundo.

Luego se evaluó el resto de parámetros de inclusión en cada una de las plataformas BPM consideradas, que se consolidó con su respectiva puntuación en la Tabla 4. 
Figura 1. Descargas de plataformas BPM en el mundo vs Porcentaje de tales descargas en Colombia entre 2015 y 2016 , según registros de repositorio de libre descarga sourceforge("sourceforge," n.d.)

Fuente: elaboración propia

\section{Cantidad Descargas Mundiales Plataformas BPM vs Porcentaje de Tales \\ Descargas en Colombia entre 2015 y 2016 obtenidos sourceforge.net}

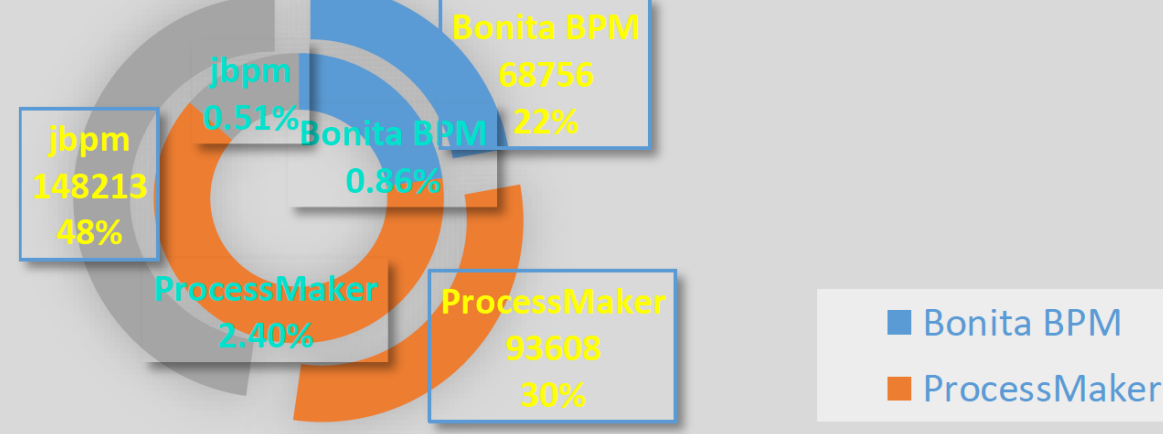

TABLA 4. RESULTADO FINAL EVALUACIÓN DE PARÁMETROS INCLUSIÓN/SELECCIÓN DE PLATAFORMAS BPM LIBRES FUENTE: ELABORACIÓN PROPIA

\begin{tabular}{c|l|c|c|c|c}
\multirow{2}{*}{ Núm. } & \multicolumn{1}{|c|}{ Parámetro Inclusión/Selección } & \multicolumn{2}{c}{ Puntuación del parámetro para el Proveedor/ } \\
\cline { 3 - 6 } & \multicolumn{1}{|c|}{$\begin{array}{c}\text { Bonitasoft } \\
\text { (Bonita } \\
\text { BPM) }\end{array}$} & ProcessMaker & $\begin{array}{c}\text { Red Hat } \\
\text { (jBPM) }\end{array}$ & $\begin{array}{c}\text { Alfresco } \\
\text { (Activiti) }\end{array}$ \\
\hline 1 & $\begin{array}{l}\text { Reconocimiento del Producto del proveedor } \\
\text { BPM como un BPMS en las publicaciones estado } \\
\text { arte. }\end{array}$ & 4 & 2 & 8 & 0 \\
\hline 2 & $\begin{array}{l}\text { Cantidad mundial descargas de la versión libre } \\
\text { de la Plataforma BPM en repositorios de libre } \\
\text { descarga }\end{array}$ & 2 & 3 & 5 & 0 \\
\hline 3 & $\begin{array}{l}\text { Porcentaje descargas de la versión libre de la } \\
\text { plataforma BPM en Colombia respecto a la } \\
\text { mundial }\end{array}$ & 4 & 5 & 1 & 0 \\
\hline 4 & $\begin{array}{l}\text { Diversidad de industrias en las que las } \\
\text { plataformas BPM del proveedor pueden ser } \\
\text { utilizadas }\end{array}$ & 4 & 4 & 0 & 2 \\
\hline 5 & $\begin{array}{l}\text { Nivel de importancia de los clientes utilizan las } \\
\text { plataformas BPM del proveedor }\end{array}$ & 4 & 5 & 0 & 0 \\
\hline 6 & $\begin{array}{l}\text { Nivel de fortaleza de la comunidad alrededor del } \\
\text { proveedor y sus plataformas BPM }\end{array}$ & 9 & 8 & 6 & 6 \\
\hline 7 & $\begin{array}{l}\text { Plataforma BPM con versión propietaria y versión } \\
\text { libre en el todo el ciclo BPM }\end{array}$ & 10 & 10 & 10 & 10 \\
\hline & TotAL & 37 & 37 & 30 & 18 \\
\hline
\end{tabular}


La evaluación evidencio que Bonitasoft y ProcessMaker obtuvieron los mejores puntajes, por lo que estas dos plataformas BPM se analizaron con los parámetros del marco de evaluación/comparación establecido en la siguiente fase del estudio.

B. Establecimiento del Marco de Parámetros de Evaluación Para Plataformas BPM (BPMS/iBPMS)

Para obtenerlos se analizaron y consolidaron las observaciones e ideas de las publicaciones y literatura considerada en el análisis, incluidos las de Forrester y Gartner mencionadas antes, referidos como Cuadrantes Mágicos de Sistemas de Gestión de Procesos de Negocio(Sinur \& Hill, 2010), enfocadas en evaluar BPMS y su capacidad para posibilitar la mejora/ optimización continua del proceso y participación activa de los roles de TI y de negocio en el ciclo de vida del proceso, y Cuadrantes Mágicos de Sistemas de Gestión de Procesos de Negocio Inteligente(Hill et al., 2012)(Schulte \& Cantara, 2014)(Dunie, Rob;Schulte, 2015) direccionados a evaluar iBPMS en cuanto a su capacidad para soportar Operaciones de Negocio Inteligente(IBO), que se refiere a integración de analítica avanzada a los procesos para mejor toma de decisiones. Para entender el enfoque de las publicaciones fue importante considerar que los iBPMS son evolución de los BPMS y ofrecen capacidades de los BPMS tradicionales y además IBO.

1) Establecimiento de Las Categorías y Subcategorías de Clasificación de los Parámetros del Marco de Comparación de una Plataforma BPM (BPMS/iBPMS). Se establecieron unas categorías y subcategorías de evaluación para clasificar los parámetros del marco de evaluación construidas con base a la identificación de los elementos esenciales en un BPMS/iBPMS, casos de uso de las plataformas BPM en la literatura analizada, las fases del ciclo BPM y la consolidación de los parámetros a incluir en el marco de evaluación obtenidos de la literatura analizada. Las categorías y subcategorías establecidas fueron:

- Estrategia BPM: Planeación, Programas BPM, Metodología BPM
- Definición de Proceso: Análisis de Proceso

- Diseño/Modelado: Composición de aplicaciones, diagramación, diseñador, Gestión de Cambios, Integración, Integración analítica, interacciones.

- Implementación/Ejecución: Ejecución, Sincronización, analítica activa, analítica sobredemanda, casos, reglas.

- Monitoreo/Medición/Respuesta: Analítica/ procesamiento eventos simples, procesamiento de eventos complejos, caso de negocio, reportes personalizados.

- Optimización/Innovación: Mejora procesos/ analítica, Mejora automática procesos, analítica predictiva,

- Gestión Recursos/Servicios involucrados: Movilidad, visibilidad, interacciones humanas, coordinación/control recursos, artefactos proceso, información procesos, errores/excepciones.

- Integración: Gestores información, conectividad, tecnologías componentes, servicios analíticas, desarrollo de software, arquitectura orientada a servicios, Internet de las cosas,

- Plataforma (aspectos técnicos): Usabilidad, Experiencia de usuario, permisos, dependencias, administración de la plataforma, innovación, tipos instalación, versiones, proceso instalación, arquitectura, asistencia técnica.

- Documentación: disponibilidad, calidad.

- Producto (aspectos de mercado): Comunidad, mercadeo, ventas, implementaciones, costos/ licenciamiento.

- $\quad$ Proveedor (fabricante): Enfoque, consultores, finanzas, aliados, planta de empleados, socios.

2) Definición del Marco de Parámetros para la Evaluación/Comparación de Plataformas BPM (BPMS/iBPMS). Se realizó un análisis detallado y profundización de todas las explicaciones, comentarios, observaciones, notas de evaluación de los artículos Gartner, Forrester y las demás referenciados, 
acerca de los diferentes proveedores de plataformas BPM analizados por ellos en tales publicaciones, para identificar, filtrar, interceptar, categorizar, consolidar y profundizar unos parámetros explícitos en un marco de evaluación claro y enfocado a nuestra meta. Esto dio como resultado 126 parámetros clasificados en las categorías y subcategorías anteriores, luego se filtraron descartando los parámetros que por tiempo y en el contexto de evaluación limitado a un análisis académico en un entorno de pruebas no nos permitiría evaluarlos correctamente, dando como resultado 85 parámetros clasificados en las categorías y subcategorías decantadas.

El resultado del análisis, consolidación y profundización de las publicaciones fue un marco de evaluación claro. Este marco categoriza y define unos parámetros explícitos deseados en una plataforma/proveedor BPM (BPMS/iBPMS). El resultado no discriminó cuales parámetros aplican para BPMS y cuales para iBPMS partiendo de la base que muchas capacidades como simulación, optimización, monitoreo de actividades de negocio e inteligencia de negocios han estado presentes por años en los
BPMS, y siguen presentes en iBPMS, pero estos han agregado soporte mejorado con tecnologías más avanzadas para colaboración humana, integración con medios sociales, acceso móvil, analítica y gestión de decisiones en tiempo real.

\section{Definición del Método de Evalua-} ción/Comparación de las Dos Plataformas BPM Seleccionadas (BPMS/iBPMS)

La evaluación de los 85 parámetros del marco de evaluación/comparación para las plataformas seleccionadas, BonitaBPM y ProcessMaker incluyo la especificación de mecanismos de evaluación (como) y criterios y rangos de calificación (cuanto).

1) Definición mecanismos para la evaluación del marco de parámetros en las dos plataformas BPM. Se partió de que no todo se puede comprobar a través de funcionalidad porque se requería de un escenario real para comprobarlos y/o medir el grado de satisfacción en muchos de los parámetros seleccionados y el contexto académico estuvo limitado a un entorno de pruebas, por lo que se definieron tres mecanismos posibles para evaluar las herramientas.

TABLA 5. MECANISMOS PARA EVALUAR LOS PARÁMETROS DEL MARCO DE EVALUACIÓN EN LOS DOS BPMS FUENTE: ELABORACIÓN PROPIA

\begin{tabular}{|c|c|c|c|}
\hline Número & $\begin{array}{c}\text { Mecanismo } \\
\text { (fuente) }\end{array}$ & Descripción & Referencias \\
\hline 1 & Documentación & $\begin{array}{l}\text { La página corporativa del proveedor, garantía } \\
\text { de ser una fuente valida, sirvió para comprobar } \\
\text { si la plataforma tiene soporte o no a determi- } \\
\text { nadas características y en qué grado puede ser } \\
\text { soportada. }\end{array}$ & $\begin{array}{l}\text { Documentación } \\
\text { - } \text { Bonita BPM documentación } \\
\text { (Bonitasoft, n.d.) } \\
\text { - } \begin{array}{l}\text { ProcessMaker documentación } \\
\text { (ProcessMaker, n.d.) }\end{array}\end{array}$ \\
\hline 2 & $\begin{array}{l}\text { Ejemplos/ } \\
\text { Recursos }\end{array}$ & $\begin{array}{l}\text { Páginas corporativas de plataformas se encon- } \\
\text { traron ejemplos prácticos, videos y documen- } \\
\text { tos con explicaciones funcionalidades de la } \\
\text { plataforma que se utilizaron para determinar el } \\
\text { soporte o grado de satisfacción de una funcio- } \\
\text { nalidad específica. }\end{array}$ & $\begin{array}{l}\text { Recursos: } \\
\text { - } \quad \text { Bonita BPM documentación } \\
\text { (Bonitasoft, n.d.) } \\
\text { - } \begin{array}{l}\text { ProcessMaker documentación } \\
\text { (ProcessMaker, n.d.) }\end{array}\end{array}$ \\
\hline 3 & $\begin{array}{l}\text { Pruebas de funcio- } \\
\text { nalidad en un pro- } \\
\text { ceso cualquiera, o el } \\
\text { de solicitud de apro- } \\
\text { bación de crédito }\end{array}$ & $\begin{array}{l}\text { Con el prototipo de un proceso de proceso de } \\
\text { solicitud de crédito que incluye todo el proceso } \\
\text { de forma que por partes nos sirva para com- } \\
\text { probar algunos de los parámetros del marco de } \\
\text { evaluación según las necesidades en el proceso. }\end{array}$ & $\begin{array}{l}\text { El proceso se modelara en la si- } \\
\text { guientes secciones y consiste en } \\
\text { un proceso completo de solicitud } \\
\text { y aprobación de crédito genérico. }\end{array}$ \\
\hline
\end{tabular}


TABLA 6. CRITERIOS CALIFICACIÓN PARÁMETROS PARA PLATAFORMAS BPM SELECCIONADAS

FUENTE: ELABORACIÓN PROPIA

\begin{tabular}{c|c|l} 
Número & Puntos & \multicolumn{1}{|c}{$\begin{array}{c}\text { Criterio para asignación de } \\
\text { puntos }\end{array}$} \\
\hline 1 & 0 & No soporta el parámetro \\
\hline 2 & 4 & $\begin{array}{l}\text { Soporta de manera básica el } \\
\text { parámetro }\end{array}$ \\
\hline 3 & 6 & $\begin{array}{l}\text { Soporta el parámetro de forma } \\
\text { intermedia }\end{array}$ \\
\hline 4 & 8 & $\begin{array}{l}\text { Soporta el parámetro de buena } \\
\text { forma }\end{array}$ \\
\hline 5 & 10 & $\begin{array}{l}\text { Soporta el parámetro de forma } \\
\text { excelente }\end{array}$ \\
\hline
\end{tabular}

2) Criterios y Rangos Calificación Parámetros a Evaluar en Dos Plataformas BPM Seleccionadas.Los parámetros se calificaron en un rango de 0 a 10 según su cumplimento (tabla VI), evaluadas utilizando combinaciones de los mecanismos descritos. A los parámetros se le dio la misma ponderación partiendo del principio que están en fases con una importancia equivalente, en especial modelado, diseño, ejecución y optimización que son las fases principales del ciclo de vida BPM, las demás fases son complementos, pero relacionadas a cada una de tales fases.

3) Proceso de Negocio Considerado en el Estudio Para Evaluar y Desplegar como Prototipo. Se seleccionó un proceso de solicitud de crédito de una entidad real que se describió así: Un cliente solicita a la entidad una cotización un crédito por un valor, esta se diligencia en un sistema de información Web por parte de un funcionario que estima/comprueba el valor de la garantía, emite el concepto acerca de la validez de las referencias, calcula valor máximo a prestar, capacidad de pago para calificar el comportamiento crediticio, emite un concepto de visita domiciliaria en caso de ser necesaria y envía la solicitud a un comité de crédito para que se analice y emita un concepto de aprobación del crédito. Tal comité se apoya en los valores calculados y los conceptos emitidos durante el proceso para tomar la decisión de si aprobar o rechazar el crédito. Finalmente se informa al analista de crédito del concepto y este informa al cliente solicitante.

El proceso completo se modelo en la plataforma Bonita BPM (ver Figura 2), mostrando las actividades e interacción entre los actores que intervienen en el proceso (analista de crédito, analista jurídico, comité de crédito, cliente).

\section{Evaluación/Comparación de las Dos Plataformas BPM Libres Seleccionadas}

Seleccionadas las dos plataformas BPM libres se aplicó el marco de evaluación ajustado al escenario de pruebas del análisis (85 parámetros). Se evaluó Bonita BPM y ProcessMaker en su versión libre más reciente a la fecha de evaluación (2016/08/30), asignando una calificación sustentada en los criterios (ver Tabla 6) y utilizando cualquiera de los mecanismos de evaluación establecidos (ver Tabla 5). El resultado fue una evaluación comparativa de los parámetros de las dos plataformas BPM. La evaluación se muestra totalizada por plataforma (Tabla 7) y categorías y subcategorías (ver Tabla 8).

TABLA 7. RESULTADO TOTALIZADO EVALUACIÓN PLATAFORMAS BPM LIBRES EN MARCO DE EVALUACIÓN/COMPARACIÓN

FUENTE: ELABORACIÓN PROPIA

\begin{tabular}{c|c|c}
\multirow{2}{*}{$\begin{array}{l}\text { Calificación acumulada de } 85 \\
\text { parámetros evaluados con un puntaje } \\
\text { máximo de 850 puntos(10 por c/u) }\end{array}$} & Bonita BPM Community Edition & ProcessMaker Open Source \\
\cline { 2 - 3 } & Version 7.3.1 x64 & Version 3.0.1 x64 \\
\hline \multicolumn{1}{c|}{ Calificación Total } & 421 puntos & 380 puntos \\
\hline
\end{tabular}




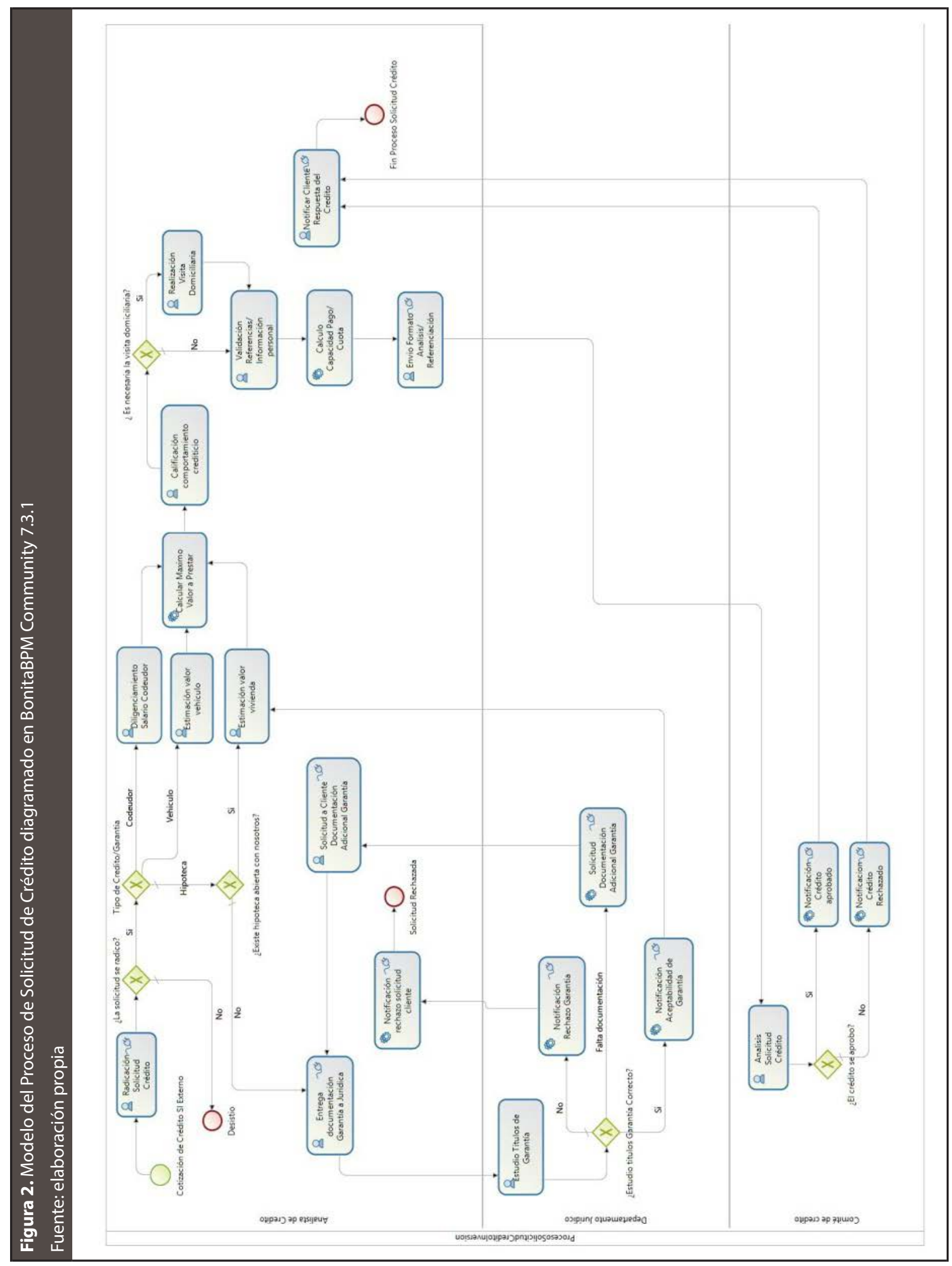


TABLA 8. RESULTADO EVALUACIÓN BPMS LIBRES EN EL MARCO DE COMPARACIÓN POR CATEGORÍASY SUBCATEGORÍAS FUENTE: ELABORACIÓN PROPIA




TABLA 9. SOFTWARE INSTALADO EN ENTORNO DE PRUEBAS PARA CONSTRUCCIÓN/DESPLIEGUE DEL PROCESO CRÉDITO FUENTE: ELABORACIÓN PROPIA

\begin{tabular}{c|l|c|l|} 
Núm. & \multicolumn{1}{|c|}{ Software } & Versión & \multicolumn{1}{c|}{ Observación } \\
\hline 1 & $\begin{array}{l}\text { Sistema Operativo } \\
\text { Windows e IIS }\end{array}$ & 8.1 & $\begin{array}{l}\text { IIS para desplegar una aplicación web con la que se integrara el } \\
\text { proceso. }\end{array}$ \\
\hline 2 & $\begin{array}{l}\text { Motor de Base de } \\
\text { datos SQL Server }\end{array}$ & 2012 & $\begin{array}{l}\text { El proceso de solicitud de crédito se integra con una aplicación web } \\
\text { que se conecta con base de datos SQL Server con información de } \\
\text { créditos. }\end{array}$ \\
\hline 3 & $\begin{array}{l}\text { Aplicación Web } \\
\text { Crédito ASP.NET }\end{array}$ & $\begin{array}{c}\text { Framework } \\
4.0\end{array}$ & $\begin{array}{l}\text { Desplegada en el IIS. La aplicación permite realizar el registro inicial de } \\
\text { la toda la información de la solicitud de crédito. }\end{array}$ \\
\hline 4 & $\begin{array}{l}\text { BonitaBPM } \\
\text { Community } \\
\text { Edition }\end{array}$ & $\begin{array}{l}\text { - BonitaStudio: Herramienta construcción de aplicación de procesos. } \\
\text { - } \begin{array}{l}\text { Motor Procesos Bonita: Componente para despliegue de procesos. } \\
\text { Servidor Web Apache Tomcat 7.0.67: Para desplegar el portal Bonita. }\end{array} \\
\text { - } \begin{array}{l}\text { Bonita BPM Portal: Para la gestión de las instancias, tares de } \\
\text { procesos. } \\
\text { Base de datos H2 1.3.170: Base de datos multiplataforma relacional } \\
\text { para almacenamiento y gestión de información de procesos. }\end{array}\end{array}$ \\
\hline
\end{tabular}

\section{Figura 3. Realización de radicado de la solicitud de crédito desde el Portal Bonita BPM por parte del analista de crédito} Fuente: elaboración propia

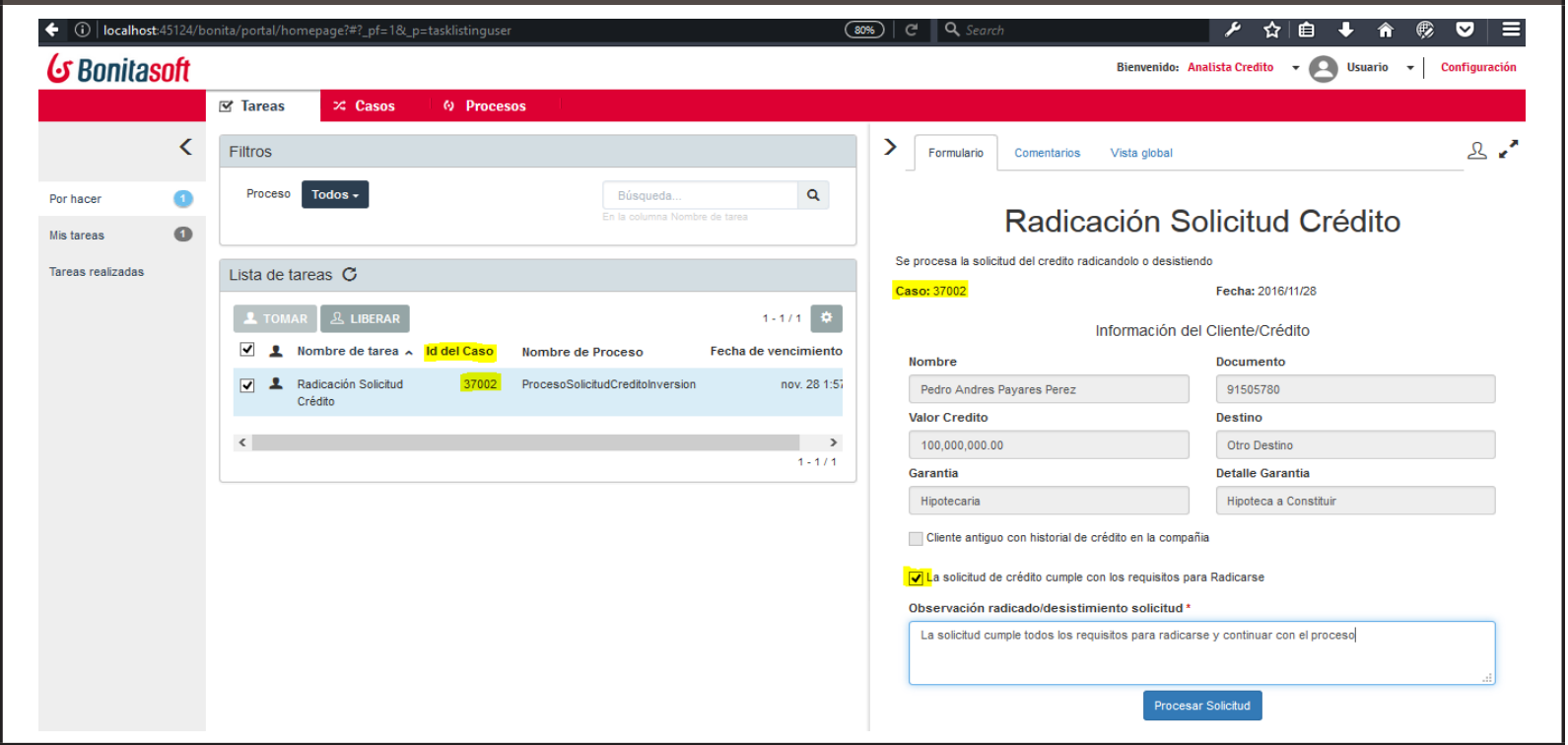

La evaluación de las dos plataformas BPM libres analizadas se resumió en la Tabla 8 acumulada por categorías y subcategorías. Esta permitió ver las pocas diferencias, aunque con una diferencia muy marcada en algunas subcategorías. La plataforma con mejor calificación fue Bonita BPM que fue utilizada para desplegar el prototipo del proceso de solicitud de crédito.

\section{E. Construcción/Despliegue de Pro-} totipo Proceso de Solicitud de Crédito en la Plataforma BONITABPM

Se modelo el proceso de solicitud de crédito (ver Figura 2), y se adecuo un entorno de pruebas para construir y desplegarlo. 
1) Adecuación del entorno de pruebas para construcción y despliegue del proceso.

Se describe el entorno de pruebas configurado (ver Tabla 9), incluida la aplicación web externa relacionada con el proceso.

2) Construcción de la aplicación de proceso en BonitaStudio de Bonita BPM

En el componente BonitaStudio de Bonita BPM se construyó la aplicación de procesos con las respectivas sendas para cada uno de los actores, analista de negocio, analista jurídico y comité de crédito y la configuración de las actividades que le correspondían a cada uno. Una instancia del proceso inicia un caso en Bonita con un evento externo de una cotización de crédito que se da en una aplicación web que utiliza el REST API ofrecido por la plataforma Bonita BPM. Iniciado el proceso, el analista de crédito radica la solicitud si el cliente cumple con todos los requisitos (ver Figura 3).

Radicada la solicitud la aplicación del proceso se actualiza el radicado en la base de datos de la aplicación web, para continuar con la ejecución del resto de las actividades de la solicitud de crédito por parte de todos los actores involucrados como se mostró en la Figura 2, para finalmente dar un concepto de aprobación o rechazo de la solicitud por parte del comité de crédito e informar al cliente vía correo electrónico desde la aplicación del proceso. La plataforma fue totalmente funcional, desplegando todo el proceso como se planteó y comprobando en detalle la evaluación realizada en varios de los parámetros evaluados, sin embargo la curva de aprendizaje fue un poco alta.

\section{Conclusiones}

El estudio entrego como principal resultado un marco solido de parámetros de evaluación/comparación con un amplio alcance para evaluar/seleccionar una plataforma software BPM dependiendo de la necesidad y funcionalidad esperadas, no solo para las versiones libres, también para las versiones propietarias, porque el establecimiento de parámetros del marco de evaluación incluyo todo el espectro de funcionalidades encontrados en todo el análisis, indiferente del tipo de pago de las plataformas.

Respecto a las plataformas software BPM libres, objeto principal del análisis son sin duda herramientas que permiten a cualquier organización/ persona iniciar en la disciplina BPM y madurar en el tema, como se demostró con el despliegue del proceso de solicitud de crédito, que nos permitió comprobar prestaciones tanto en BonitaBPM como en ProcessMaker. Sin embargo, el espectro de funcionalidades es muy amplio y difícilmente al iniciar en BPM se va a requerir todo el conjunto de funcionalidades posibles, esto requiere de un proceso de maduración a nivel de todos los recursos involucrados en el despliegue de procesos a nivel organizacional.

En cuanto al alcance de una plataforma software BPM libre con la experiencia del análisis, específicamente con la realización del prototipo del proceso, es claro que en una implementación de BPM en una organización, a medida que despliegue más procesos, más actores se involucren y se integren todas las aplicaciones empresariales subyacentes a tales procesos, mantenerse en la versión libre será más costoso que pasarse a las versiones pagas o comprar una plataforma BPM propietaria, por el tiempo y la cantidad de recursos que habría que invertir para mantener, soportar y ampliar la cobertura de los procesos con la plataforma BPM en un ambiente de producción, pero sin duda las versiones libres son una opción para explorar e iniciar en el tema BPM.

En el análisis se infirieron una serie de conclusiones puntuales acerca de las plataformas BPM libres analizadas, importantes en un contexto BPM, en cualquier organización:

- $\quad$ Están más inclinadas al desarrollador que al analista de negocio, al menos en la fase de construcción y administración de las aplicaciones de procesos, aspecto contrario al manejo de los casos por los actores asignados en donde el esquema permite que cualquiera pueda atender los casos a través de un portal. 
- $\quad$ La curva de aprendizaje es alta y más inclinada a medida que se sube la complejidad porque no está soportada por una comunidad tan grande que permita resolver dudas de nivel intermedio o avanzado y o inconvenientes ni tan rápido ni tan efectivamente, para eso los proveedores de plataformas cuentan con paquetes de formación, o están sujetos a las versiones pagas de la plataforma, por lo que se desvirtúa el tema de no costo normalmente asociado a lo libre en un entorno de producción en el que se necesitan respuestas rápidas, que solo se logra con soporte y/o asesoría.

- El resultado de la evaluación en el marco de parámetros en sus versiones libres, a la fecha BonitaBPM, a pesar de obtener mejor calificación comparada con ProcessMaker, en muchos de los parámetros las diferencias son menores e incluso esta última supera a BonitaBPM en algunos de los parámetros, por lo que sin duda, también es una buena opción para iniciar en el tema BPM.

- $\quad$ El prototipo del proceso de solicitud de crédito se desplego, sin embargo, para lograr el objetivo del proceso y las interacciones requeridas entre los actores el nivel de dificultad fue alta y requirió en muchas partes del proceso aplicar la técnica de fuerza bruta para encontrar la solución y/o comportamiento deseado con base en que la documentación o recursos, en muchos casos son muy superficiales, incluso en funcionalidades sencillas, lo que se explica en que proveedores de plataformas BPM como Bonita/ProcessMaker sustentan su negocio en buena parte en paquetes de formación que sin duda se requerirán en una implementación real de BPM con tales plataformas, por lo que el costo más en que en la licencia, realmente está en la formación, soporte y asesoría. Aspecto importante a tener en cuenta al momento de decidir implementar BPM con una plataforma BPM libre.

\section{Referencias}

Activiti. (n.d.). Retrieved from http://activiti.org/

Binner, H;Bariff, M;Benedict, T; Champlin, B; Downing, B; Franzen, J. (2009). Guide to the Business Process Management Common Body of Knowledge: ABPMP BPM $C B O K ®$. Association of Business Process Management Professionals.

Bonitasoft. (n.d.). Bonita BPM Documentation. Retrieved from http://documentation.bonitasoft.com/

BPM-Latino.com. (n.d.). Retrieved from http://www.bpmspain.com/

Brocke, J; Rosemann, M. (2010). Handbook on Business Process Management: Strategic Alignment, Governance, People and Culture (Vol. 1). International Handbooks on Information Systems.

Club-BPM. (n.d.). Retrieved from http://www.club-bpm. com/CasosExito.htm

Dunie, Rob;Schulte, R. M. M. (2015). Magic Quadrant for Intelligent Business Process Management Suites. Gartner Inc.

Harmon, P. (2016). The State of Business Process Management 2016. BPTrends

Hill, J. B., Jones, T., Schulte, W. R., \& Sinur, J. (2012). Magic Quadrant for Intelligent Business Process Management Suites. Gartner Inc, (September), 1-33.

Histpass, B. (2014). Business Process Management (BPM) Fundamentos y Conceptos de Implementación (Tercera Ed). Santiago de Chile: BPM Center.

Hitpass, B. (2014). Gestión de Procesos de Negocio - Fundamentos y Conceptos de Implementación (Tercera).

Kitson, N., Ravisanskar, R., \& Soudamini, R. N. (2012). Case Management - Managing chaos: unstructured processes and dynamic BPM. Capgemini, 1-8. Retrieved from www.capgemini.com/bpm-trends

Miers, D. (2013). The Forrester Wave: BPM Suites, Q1 2013. Forrester Research, Inc., 1-21.

Moore, C;Saxena, Raju;Lee, D. E. (2013). Guide to the Business Process Management Common Body of Knowledge BPM-CBOK. Association of Business Process Management Professionals.

ProcessMaker. (n.d.). ProcessMaker BPM documentation. Retrieved from http://wiki.processmaker.com/index.php/Main_Page 
Schulte, W. R., \& Cantara, M. (2014). Magic Quadrant for Intelligent Business Process Management Suites. Gartner Inc, (March).

Sinur, J., \& Hill, J. B. (2010). Magic Quadrant for Business Process Management Suites, (October).

sourceforge. (n.d.). Retrieved from https://sourceforge. net/ 